\title{
DIE WIRKUNG VON HUMUSSTOFFEN AUF DEN PFLANZLICHEN STOFFWECHSEL
}

\author{
W. FLAIG \\ Aus dem Institut für Biochemie des Bodens der Forschungsanstalt für \\ Landwirtschaft Braunschweig-Völkenrode
}

Eingegangen am 20. 10. 1960.

Ein Teil der organischen Stoffe im Boden erreicht im Verlauf ihrer Umwandlung eine verhältnismässig stabile Zwischenstufe. Diese ist derjenige Teil der organischen Substanz, den man als Humusstoffe bezeichnet. Die verhältnismässig beständigen Humusstoffe haben besondere Aufgaben im Zusammenhang mit dem Pflanzenwachstum.

Ihre Beteiligung an den Vorgängen kann auf zweierlei Weise erfolgen. Einmal erlangen sie auf Grund ihrer physikalischen Eigenschaften eine Bedeutung, zum anderen beteiligen sich die Abbauprodukte bei den chemischen Umsetzungen der verschiedenen Bodenbestandteile und können den Stoffwechsel der Pflanzen direkt beeinflussen, indem sie von den Pflanzen aufgenommen werden. Die hochmolekularen Substanzen der organischen Stoffe im Boden haben hauptsächlich aufgrund ihrer physikalischen Eigenschaften einen Effekt auf das Pflanzenwachstum, während die niedermolekularen einen grösseren Einfluss auf die chemischen und biologischen Vorgänge besitzen, indem sie z.B. in den Stoffwechsel der Pflanzen eingreifen.

Auf die Bedeutung der organischen Substanzen des Bodens im Hinblick auf deren physikalische Eigenschaften soll nur kurz und auch nur im Zusammenhang mit deren Einfluss auf das Pflanzenwachstum eingegangen werden.

Tab. 1. Anteil der anorganischen und organischen Substanz an der Sorption (Schachtschabel)

\begin{tabular}{|c|c|c|c|c|c|}
\hline & $\begin{array}{c}\text { Humus } \\
\%\end{array}$ & $\begin{array}{l}\text { T-Wert } \\
\text { Gesamt- } \\
\text { Boden }\end{array}$ & $\begin{array}{l}\text { (mval } 100 \mathrm{~g} \\
\text { anorg. } \\
\text { Anteil }\end{array}$ & $\begin{array}{l}\text { Boden) } \\
\text { org. } \\
\text { Anteil }\end{array}$ & $\begin{array}{c}\text { Anteil } \\
\text { des Humus } \\
\text { am T-Wert } \\
\text { in } \%\end{array}$ \\
\hline Heidesand & 4,25 & 9,1 & 2,5 & 6,6 & 73 \\
\hline Buntsandstein & 1,44 & 4,3 & 2,3 & 2,0 & 46 \\
\hline Kulmtonschiefer & 5,78 & 17,5 & 9,2 & 8,3 & 47 \\
\hline Lösslehm & 3,20 & 19,3 & 11,3 & 8,0 & 41 \\
\hline Rötton & 3,37 & 24,7 & 16,1 & 8,6 & 35 \\
\hline
\end{tabular}


Nach Untersuchungen von Schachtschabel (37) und anderen sind die organischen Stoffe zwar nur zu wenigen Prozenten im Boden vorhanden, beteiligen sich aber durchschnittlich zu $50 \%$ an dessen Sorptionskapazität. Den organischen Stoffen kommt daher als Austauscher eine grosse Bedeutung für die Regulierung des Nährstoffhaushaltes zu.

Der physikalische Effekt der hochmolekularen Substanzen auf das Pflanzenwachstum kann in Beziehung zu der Grösse und der Form ihrer Moleküle gebracht werden. Sie können einen Einfluss auf die Grösse der Durchmesser und die Stabilität der Bodenkrümel haben. $\mathrm{Zu}$ den organischen Bodenkolloiden gehören Proteine, Polysaccharide, Polyuronide und die Gruppe der Huminsäuren. Darunter befinden sich sowohl Sphäro- wie auch Linearkolloide. Das Verhalten dieser beiden Arten von Kolloiden ist sehr unterschiedlich. Als ein Beispiel für Sphärokolloide sollen Huminsäuren erwähnt werden.

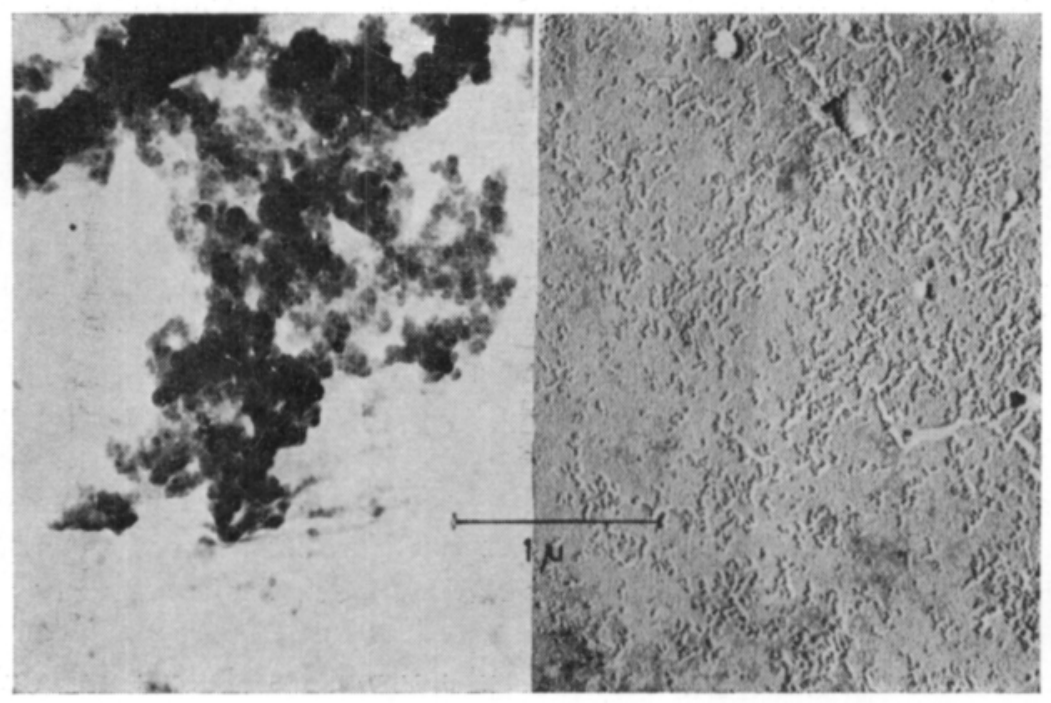

Abb. 1. Elektronenmikroskopische Aufnahmen von Schwarzerdehuminsäuren $\mathrm{pH}=\mathbf{3}$ und $\mathrm{pH}=\mathbf{8}$

Der Durchmesser dieser Sphärokolloide ist zwischen 80 und $100 \AA(1,11,12)$. In saurer Lösung aggregieren die Teilchen, in alkalischer Lösung werden die Aggregate dispergiert. Verschiedene physikalische Untersuchungen stützen die Annahme, dass die Bindung zwischen den einzelnen Teilchen über Wasserstoffbrücken erfolgt.

Einige Eigenschaften der Linearkolloide sind beträchtlich verschieden von denen der Sphärokolloide. So ist bekannt, dass die Linearkolloide selbst in sehr geringen Mengen die Viskosität ihrer Lösungen erhöhen. Heteropolare Linearkolloide, wie Polyuronsäuren oder Krilium zeigen ein sehr unterschiedliches Verhalten von dem der heteropolaren Sphärokolloide wie Huminsäuren. Je nach dem Dissoziationsgrad findet bei den Linearkolloiden eine Knäuelung oder eine Streckung der fadenförmigen Moleküle statt. 
Im Zusammenhang mit anderen Untersuchungen macht man die Beobachtung, dass die Linearkolloide einen viel stärkeren Effekt auf die Flockung von Tonmineralen haben als die Sphärokolloide. Aus diesem Grunde hat man auch versucht, synthetische Linearkolloide wie Krilium als Bodenverbesserungsmittel anzuwenden. Die Vorgänge der Flockung von anorganischen Kolloiden, insbesondere die der Tonminerale durch heteropolare Linearkolloide, ist ein sehrkomplexer Vorgang (24).

Die physikalischen Bedingungen für das Pflanzenwachstum sind eng mit einer bestimmten Grösse der Bodenkrümel verbunden. Diese beeinflussen die Nachlieferung der pflanzlichen Nährstoffe, den Wasser- und Lufthaushalt und sind damit einer der wichtigsten Faktoren für die Bodenfruchtbarkeít.

Nach HAGIN (26) ist z.B. der Ertrag von Roggenkeimpflanzen $(20,1 \pm 0.7 \mathrm{~g})$ am höchsten, wenn mehr als $90 \%$ der Krümel einen grösseren Durchmesser als $1 \mathrm{~mm}$ haben. Sind mehr als $80 \%$ der Krümel kleiner als $0,5 \mathrm{~mm}$, so ist die Struktur ungünstig und man erhält nur einen sehr niedrigen Ertrag von $5.8 \pm 0.7 \mathrm{~g}$.

Diese Übersicht über die physikalische Beeinflussung des Pflanzenwachstums durch Humusstoffe möge genügen. Dabei soll als wesentlichste Tatsache hervorgehoben werden, dass bei dem physikalischen Einfluss der Humusstoffe auf das Pflanzenwachstum grössere Mengen von ungefähr $1 / 100$ bis $1 \%$ des Bodengewichtes notwendig sind.

Es bestehen jedoch noch andere Effekte der Humusstoffe auf das Pflanzenwachstum, die durch einen um mehrere Zehnerpotenzen geringeren Prozentgehalt bewirkt werden. Dieses sind die chemischen oder physiologischen Einwirkungen auf den Stoffwechsel der Pflanzen. Die physikalische Wirkung der Humusstoffe auf das Pflanzenwachstum ist schon ein komplexer Vorgang, es scheint jedoch so zu sein, dass die chemischen oder physiologischen Wirkungen noch komplizierter sind. Verschiedene Wirkungen des Humus beruhen weder auf einem Nährstoffeinfluss, noch sind sie durch die Einwirkung auf physikalische Bodeneigenschaften zu erklären. Dieser, in der landwirtschaftlichen Literatur als "reine Humuswirkung" (39) angesprochene Einfluss kann nur darauf zurückgeführt werden, dass Stoffe aus dem Humus den Stoffwechsel der Pflanze beeinflussen.

Beim Abbau der organischen Stoffe im Boden tritt eine Wirkung auf das Pflanzenwachstum auf, die im Zusammenhang mit der Verfügbarkeit der Phosphorsäure und und des Kaliums stehen.

Fuller und Nielsen (25) stellten fest, dass die Verfügbarkeit der im Boden vorhandenen Phosphorsäure durch unverrottete organische Stoffe wie z.B. Stroh mehr erhöht wird als durch Strohkompost. Es sind somit die Abbauprozesse im Boden für die günstigere Phosphorsäureversorgung verantwortlich. zu ähnlichen Befunden kommen auch Dalton, Russel und Sieling (6).

SaUERLANDT und GrafF (35) konnten im Feldversuch nachweisen, dass durch Strohgaben die Gehalte an laktatlöslicher Phosphorsäure und Kali erhöht werden.

Da durch die Rotte verschiedenster organischer Stoff im Boden die pflanzenverfügbaren Gehalte an Kalium und Phosphorsäure erhöht werden, liegt die Annahme nahe, dass durch biologische Abbauprozesse im Boden ganz allgemein die Kali- und Phosphorsaureversorgung der Pflanzen begünstigt wird. 
Hiervon seien einige Versuche erwähnt (20). Eine Mischung von Schwarzerde und Sand wurde mit einer Nährlösung versetzt. Einem Teil davon wurden zwei verschieden hohe Mengen ( 0.6 und $1.2 \%$ ) an gehäckseltem Stroh zugesetzt. Eine Probe des Boden-Sand-Gemisches und die beiden mit Stroh versetzten wurden nach 70 Tagen Bebrütung bei $28^{\circ}$ und $90 \%$ relativer Luftfeuchtigkeit gemahlen und im Verhältnis 1:2,5 wiederum mit Quarzsand vermischt. Nach Anfeuchten mit destilliertem Wasser wurde mit Sommerroggen bepflanzt und die Keimpflanzen nach 14 Tagen geerntet. Es ergab sich bei der niederen Strohgabe eine gesicherte Erhöhung des Sprosstrockengewichtes und bei der höheren eine solche von Sprossund Wurzeltrockengewicht.

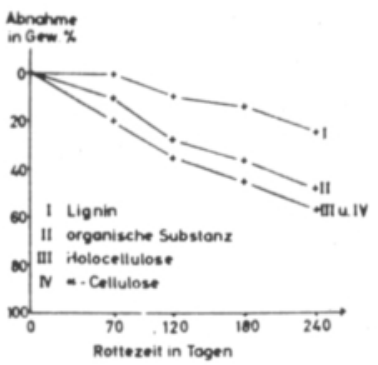

D1e Abnahme von organischer Substanz, Lignin, Holound L-Cellulose der Variante ohne St1ckstoffzusata zur Rotte (Die Prozente sind auf die jewelligen Ausgangewerte bezogen)
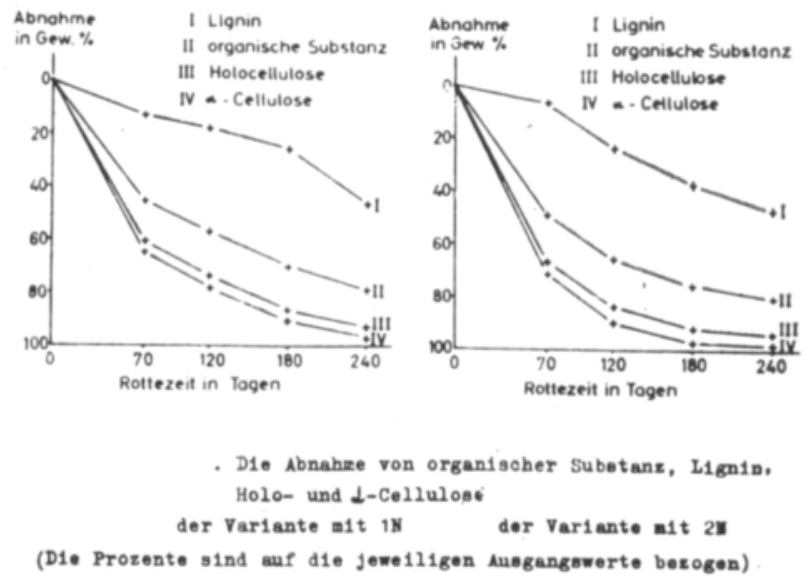

Abb. 2.

Die Analysen der Pflanzen zeigten, dass die aufgenommenen Mengen an P und K sowie auch an Fe erhöht wurden. Auch der Prozentgehalt dieser Nährstoffe erfuhr eine Erhöhung.

Die geringere Aufnahme an Stickstoff bei der höheren Strohgabe kann darauf zurückgeführt werden, dass nach 70 Tagen die biologische Umsetzung des zuge- 
setzten Strohs noch nicht vollständig abgeschlossen war, und der Stickstoff immobilisiert wurde.

Ferner konnte festgestellt werden, dass der Gehalt an laktatlöslicher Phosphorsäure zugenommen hat. Nach der Methode von Sauerlandt, Scheffer und Banse (36) ist der Gehalt an wasserlöslicher anorganischer und säure- und basenlöslicher organisch gebundener Phosphorsäure ebenfalls erhöht.

Um den Einfluss verschiedener Mengen von Stickstoff auf den Verlauf der Rotte (15) zu zu überprüfen, wurde Roggenstroh in einer Klimakammer bei $29^{\circ}$ und ca. $90 \%$ iger Luftfeuchtigkeit in verschiedenen Proben verrottet. Der einen Probe wurde eine Nährlösung hinzugefügt, bei der der Stickstoff fehlte. In der zweiten Probe wurden 0,5 und in der dritten Probe $1 \%$ Stickstoff als Ammonnitrat, bezogen auf das trockene Stroh, der Nährlösung zugegeben.

Durch die verschiedenen Stickstoffgaben nahm die Verrottung der drei Proben einen unterschiedlichen Verlauf. Die Variante ohne Stickstoff (O N) war nur in einem geringen Masse abgebaut worden, während die beiden anderen grössere Veränderungen in ihrer Zusammensetzung erfuhren. Die Variante mit der doppelten Stickstoffmenge $(2 \mathrm{~N})$ befand sich schon nach 180 Tagen in einem gewissen Endzustand der Rotte. Dies war bei der Probe mit der einfachen Stickstoffmenge $(1 \mathrm{~N})$ erst nach 240 Tagen der Fall. Die Variante ohne $(\mathrm{O} \mathrm{N})$ ist am wenigsten verrottet und nach unseren Erfahrungen sind nach 240 Tagen die Abbauprozesse noch nicht zu Ende gewesen.

Mit den Proben von 240 Tagen Rottezeit sind nach Nährstoffausgleich von P und K zu einem Schwarzerde-Sand-Gemisch (1:1) Gefässversuche durchgeführt worden. In alle Gefässe wurden solche Mengen an verrottetem Stroh hinzugefägt, die in ihrem Stickstoffgehalt gleich waren. Durch die unterschiedliche Zusammensetzung und dem damit unterschiedlichen Gehalt an Stickstoff der einzelnen Rotteproben wurden Strohmengen im Verhältnis $1(2 \mathrm{~N}): 1,25(1 \mathrm{~N}): 5,5(\mathrm{O} N)$ gegeben. Ohne auf die Versuche näher einzugehen, konnte aus den erhaltenen Pflanzenerträgen geschlossen werden, dass in Abhängigkeit der Bedingungen der Rotte in dem verrottetem Stroh Substanzen vorhanden sein müssen, die das Wachstum der Versuchspflanzen günstig beeinflussen können.

Auch Chaminade (3), Winter (40), Nishita, Kowalesky und Larson (29) vermuten, dass beim Abbau pflanzlichen Materials im Boden Stoffe auftreten, die eine fördernde Wirkung auf das Pflanzenwachstum ausüben.

Im weiteren Verlauf der Untersuchungen war es von Interesse, in welchem Masse sich im Laufe der Verrottung Stoffe bilden, die einen Einfluss auf das Pflanzenwachstum ausüben können.

Tab. 2. Gesamtmenge der von den Pflanzen aufgenommenen Nährstoffe in mg; Stroh 70 Tage im Boden vorgerottet.

\begin{tabular}{ccccc}
\hline $\begin{array}{c}\text { Neubauerversuch } \\
\text { Strohgabe }\end{array}$ & Gesamt-N & $\mathrm{P}_{2} \mathrm{O}_{5}$ & $\mathrm{~K}_{2} \mathrm{O}$ & $\mathrm{Fe}$ \\
\hline $\mathrm{O}$ & 52,1 & 26,1 & 44,8 & 0,81 \\
einfach & 52,3 & 30,4 & 47,4 & 0,88 \\
doppelt & 51,2 & 32,0 & 52,4 & 0,98 \\
\hline
\end{tabular}


Unter den gewählten Bedingungen (9) der Strohrotte herrschte zwischen 30 und 80 Tagen Rottezeit die grösste biologische Aktivität. Die organische Substanz wurde zum Betriebsstoffwechsel der Mikroorganismen benötigt. Danach trat ein Stillstand des biologischen Abbaus ein und die organische Substanz nahm nach dieser Zeit kaum noch ab. Auf Grund der Änderungen der Zusammensetzung der entstehenden Produkte konnte festgestellt werden, dass jedoch noch weitere Veränderungen der Rotteprodukte durch Oxydation eintraten. Die Zeit zwischen 70 und 180 Tagen scheint am günstigsten für die Bildung wirksamer Substanzen zu sein (19).

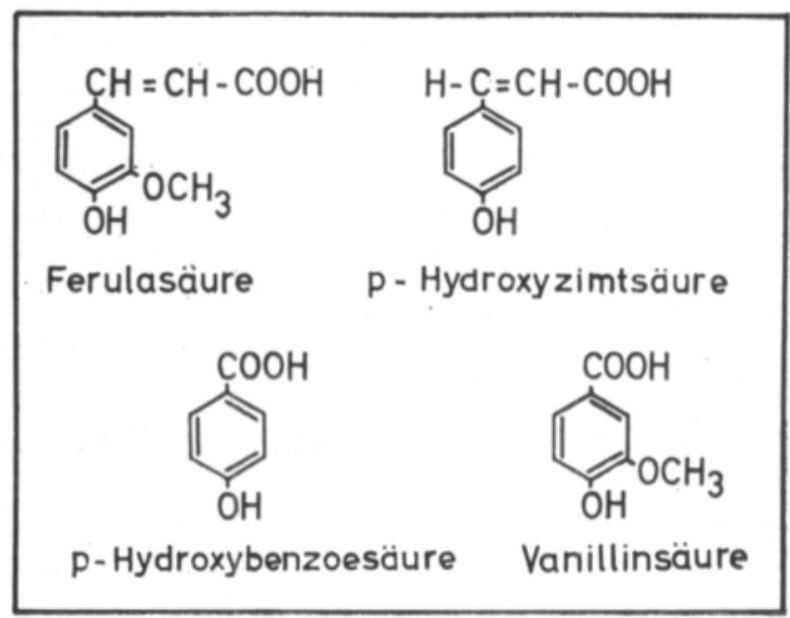

Untersuchungen von BöRNER (2) geben einen Hinweis auf die chemische $\mathrm{Zu}$ sammensetzung der Stoffe, die bei der Wirkung auf das Pflanzenwachstum beteiligt sein sein können.

Durch Extraktion von unverrottetem Stroh mit kaltem Wasser konnte er p-Hydroxyzimtsäure, Ferulasäure, p-Hydroxybenzoesäure und Vanillinsäure isolieren. p-Hydroxyzimtsäure übt in bestimmten Konzentrationen einen fördernden Einfluss auf das Anfangswachstum von Gerste aus. p-Hydroxyzimtsäure kann als Vorstufe bzw. Abbauprodukt des Lignins aufgefasst werden. Es ist daher möglich, dass diese oder ähnliche Verbindungen bei der Rotte von Stroh in solchen Mengen auftreten, dass sie das Pflanzenwachstum günstig beeinflussen können. Im Zusammenhang mit Arbeiten über den pflanzlichen Stoffwechsel war es daher von Interesse, inwieweit sich Ligninabbauprodukte auch während der Strohrotte nachweisen liessen.

Aus verschieden lange gerottetem Stroh $(70,120,180,240$ Tage), das mit Nährlösungen mit verschiedenen Mengen an Stickstoff $(0 \%, 0,5 \%, 1,0 \% \mathrm{~N}$ als $\mathrm{NH}_{4} \mathrm{NO}_{3}$ ) versetzt worden war, sind papierchromatographisch 11 verschiedene Substanzen gefunden worden, die auf Phenolnachweise positiv ansprechen. Einige von diesen Substanzen wie Vanillinsäure, Ferulasäure, p-Hydroxyzimtsäure, Vanillin und p-Hydroxybenzaldehyd konnten identifiziert werden (15). 
Es wurden daher verschiedene Ligninabbauprodukte, Fraktionen aus dem Humus und chemisch ähnliche Substanzen zunächst auf das Anfangswachstum von Getreidepflanzen überprüft.

In Neubauerschalen werden $400 \mathrm{~g}$ Quarzsand mit $70 \mathrm{ml}$ einer Knopschen Nährlösung versetzt, die die zu untersuchenden Stoffe in $10^{-4}-10^{-6}$ molarer Lösung, d.h. 2,0 bis $0,02 \mathrm{mg}$ enthielten. Die Versuche werden in einem Raum durchgeführt, dessen Beleuchtung, Temperatur und Luftfeuchtigkeit innerhalb geringer Grenzen konstant gehalten werden.

Je nach Konzentration und der chemischen Konstitution der Substanzen waren unterschiedliche Wirkung auf das Pflanzenwachstum zu beobachten. Unter günstigen Bedingungen wird eine Erhöhung der Trockengewichte von Spross und Wurzel um ca. $10-15 \%$ erhalten; die fördernde Wirkung wird durch klimatische Faktoren beeinflusst.

Mit dieser Versuchsanstellung überprüften wir nicht nur den Einfluss von Abbauprodukten des Lignins, sondern auch den von chemisch ähnlichen Substanzen auf das Wachstum, den Ertrag und die Nährstoffaufnahme von Keimpflanzen.

Tab. 3. Einfluss von p-Oxyzimtsäure, Vanillinsäure u. Ferulasäure auf das Trockengewicht von Roggenkeimpflanzen in $\mathrm{g}$.

\begin{tabular}{ccccccc}
\hline $\begin{array}{c}\text { Konzentration } \\
\text { in Mol }\end{array}$ & \multicolumn{2}{c}{ p-Oxyzimtsäure } & \multicolumn{2}{c}{ Vanillinsäure } & \multicolumn{2}{c}{ Ferulasäure } \\
& Spross & Wurzel & Spross & Wurzel & Spross & Wurzel \\
\hline 0 & 0,57 & 0,30 & 0,60 & 0,37 & 0,92 & 0,49 \\
$10^{-4}$ & 0,66 & 0,31 & 0,74 & 0,58 & 0,88 & 0,49 \\
$10^{-5}$ & 0,67 & 0,31 & 0,70 & 0,47 & 0,92 & 0,48 \\
$10^{-6}$ & 0,54 & 0,30 & 0,70 & 0,44 & 0,93 & 0,48 \\
\hline
\end{tabular}

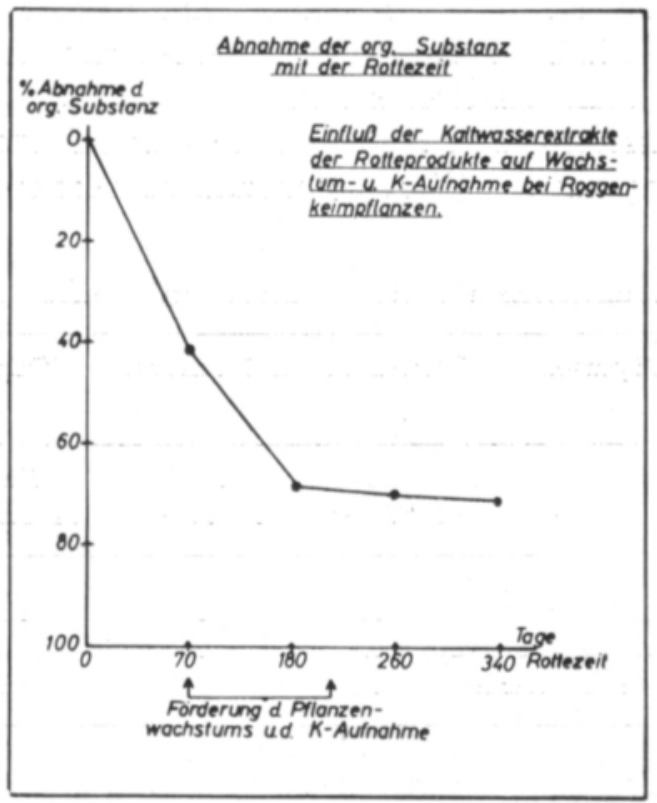

Abb. 3. Förderung des Pflanzenwachstums und der K-Aufnahme 
Während die Trockensubstanzerträge, z.B. durch p-Hydroxyzimtsäure und Vanillinsäure in Konzentrationen von $10^{-4}$ bis $10^{-5}$ molar erhöht worden sind, zeigte Ferulasäure keine Wirkung (34). Die Wirkung dieser Substanzen ist nicht immer in gleichem Masse zu beobachten, sondern hängt von einigen Faktoren ab, von denen noch nicht alle bekannt sind und von denen z.B. die Einwirkung der Luftfeuchtigkeit von uns näher untersucht worden ist.

Tab. 4. Einfluss von Huminsäuren auf das Wachstum von Mais (PANKowA).

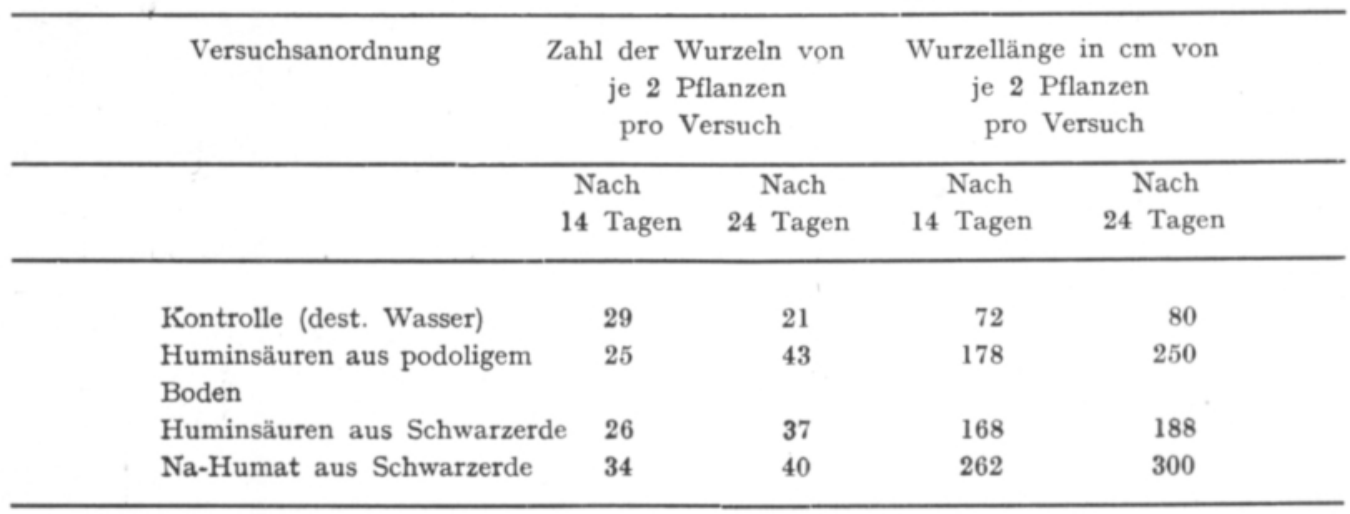

Die Art der Extraktion der Ligninabbauprodukte aus dem Stroh und die Feststellung ihres Einflusses auf das Pflanzenwachstum lassen vermuten, dass ähnliche Wirkungen auch unter natürlichen Verhältnissen auftreten können. Eine Kaltwasserextraktion des gerotteten Strohs ist vergleichbar mit dessen Auswaschung durch Niederschläge. Die Wirkung der an sich im Boden verhältnismässig instabilen Verbindungen kann damit eine Erklärung finden, dass diese aus dem Stroh über eine gewisse Zeit laufend nachgeliefert werden und dadurch einen Einfluss auf das Pflanzenwachstum ausüben können.

Auch mit Humusstoffen ist nach Kononowa und Pankowa (27) in Wasserkulturen eine Wirkung auf das Wurzelwachstum zu beobachten.

Einfluss der Humusstoffe auf das Wachstum von Weizen (PANkowA).

\begin{tabular}{lcccc}
\hline Versuchsbedingungen & Wurzelzahl bei 2 Pflanzen & $\begin{array}{c}\text { Wurzelläge in cm bei } \\
\text { 2 Pflanzen }\end{array}$ \\
\hline & $\begin{array}{c}\text { Nach } \\
\text { Tagen }\end{array}$ & $\begin{array}{c}\text { Nach } \\
\text { Tagen }\end{array}$ & $\begin{array}{c}\text { Nach } \\
\text { Tagen }\end{array}$ & $\begin{array}{c}\text { Nach } \\
\text { Tagen }\end{array}$ \\
\hline Kontrolle (dest. Wasser) & 25 & 25 & 22 & 22 \\
Huminsäuren aus Schwarzerde & 22 & 28 & 91 & 125 \\
Na-Humat aus Schwarzerde & 11 & 15 & 81 & 115 \\
\hline
\end{tabular}

Aus den angeführten Zahlen geht hervor, dass Huminsäuren sowohl auf die Vermehrung der Wurzeln als auch auf deren Längenwachstum bei Mais und Weizen 
eine Wirkung besitzen. Diese Autoren benutzen im allgemeinen Konzentrationen von Huminsäuren von $0,005 \%$ oder $5 \times 10^{-6} \mathrm{~g} / \mathrm{ccm}$.

Von Prat und Pospisil (30) konnte mit Kohlenstoff-14 markierten Humussubstanzen und von Winter und SchöNBERG (41) mit Salicylsäure nachgewiesen werden, dass diese mehr oder minder aufgenommen werden.

Wenn man Huminsäuren oder andere Fraktionen aus Humusstoffen aus verschiedenen Böden oder anderem Material isoliert, ist man nie sicher, dass die dabei erhaltenen Substanzen immer die gleiche Zusammensetzung haben. Es ist daher notwendig, Untersuchungen mit einer Reihe von chemisch ähnlichen Substanzen, sogenannten Modellsubstanzen (10), zu machen, die eine definierte chemische Konstitution haben.

Wir haben daher Untersuchungen mit Ligninabbauprodukten und verschiedenen anderen Substanzen fortgeführt, die sowohl zu jenen als auch auf Grund chemischer Untersuchungen zu Vorstufen von Huminsäuren in ihren Reaktionen eine Beziehung haben. Eine von diesen ist z.B. Thymohydrochinon. Nicht nur die Untersuchungen auf das Anfangswachstum, sondern auch Gefässversuche haben mit diesen Substanzen zu vergleichbaren Ergebnissen geführt (19).

Tab. 5. Einfluss von Protocatechusäure, Vanillin (Sommerweizen) und Thymohydrochinon (Summerroggen) auf die Erträge in Mitscherlichefässen und auf den Gehalt von $\mathrm{N}, \mathrm{P}_{2} \mathrm{O}_{5}$ und $\mathrm{K}_{2} \mathrm{O}$ in $\%$ der Trockensubstanz. Unterstrichene Werte sind statistisch gesichert.

\begin{tabular}{|c|c|c|c|c|c|c|c|c|}
\hline \multirow[b]{2}{*}{ Substanz in $10^{-5} \mathrm{Mol}$} & \multicolumn{5}{|c|}{ Korn } & \multicolumn{3}{|c|}{ Stroh } \\
\hline & rel. Ertrag & $\mathrm{N}$ & $\mathrm{P}_{2} \mathrm{O}_{5}$ & $\mathrm{~K}_{2} \mathrm{O}$ & rel.Ertrag & $\mathrm{N}$ & $\mathrm{P}_{2} \mathrm{O}_{5}$ & $\mathrm{~K}_{2} \mathrm{O}$ \\
\hline unbehandelt & 100 & 2,03 & 0,52 & 0,49 & 100 & 0,31 & 0,08 & 0,66 \\
\hline 6 Protocatechusäure & 111 & 2,03 & 0,48 & 0,61 & 104 & 0,29 & 0,08 & 0,65 \\
\hline $12 \quad$, & 110 & 0,03 & 0,60 & 0,65 & 113 & 0,31 & 0,09 & 0,73 \\
\hline 6 Vanillin & 113 & 1,88 & 0,62 & 0,71 & 108 & 0,27 & 0,09 & 0,59 \\
\hline $12 \quad$ & 111 & 2,00 & 0,52 & 0,61 & 111 & 0,29 & 0,10 & 0,51 \\
\hline unbehandelt & 100 & 2,38 & 0,50 & 0,46 & 100 & 0,67 & 0,13 & 0,49 \\
\hline 6 Thymohydrochinon & 123 & 2,07 & 0,66 & 0,62 & 106 & 0,56 & 0,13 & 0,43 \\
\hline 12 & 115 & 2,23 & 0,52 & 0,63 & 107 & 0,63 & 0,12 & 0,48 \\
\hline
\end{tabular}

Die Zusammenstellung zeigt, dass die Prozentgehalte an Stickstoff und Phosphorsäure in allen Fällen verhältnismässig geringe Unterschiede zeigen, gleichgültig, ob mit Abbauprodukten von Lignin oder Thymohydrochinon als Modellsubstanz gearbeitet worden ist. Die Kaliumgehalte sind in allen Fällen erhöht. Wie erwähnt, ist die Erhöhung der Erträge von gewissen klimatischen Bedingungen abhängig.

Im Anschluss an die Problemstellungen bei den Humusstoffen ist die Abhängigkeit der Wirksamkeit verschieden substituierter Chinone oder Hydrochinone von ihrer chemischen Konstitution untersucht worden. 
Die Wirkung dieser Verbindungen ist durch verschiedene Faktoren bedingt.

a) Es besteht eine Abhängigkeit der Wirkung von der Art, der Stellung und der Anzahl der Substituenten.

b) Chinone sind im allgemeinen in geringeren Konzentrationen wirksamer als Hydrochinone. Letztere sind meistens leichter löslich. Es müssen demnach auch physikalische Eigenschaften für die Aufnahme in die Pflanze und für den Transport an den Ort der Wirkung von Bedeutung sein.

Nach den Feststellungen auf den Ertrag begannen wir die Wirkung auf den Stoffwechsel im einzelnen zu untersuchen.

So werden die Aktivitäten verschiedener Enzyme des Zuckerstoffwechsels $(21,16)$ durch Thymohydrochinon verändert. In den Blättern von Buschbohnen z.B. in Wasserkulturen werden die Aktivitäten von Aldolase und Saccharase erhöht, während die von Amylase und Phosphatase keine deutlichen Veränderungen erfahren. Die Wirkung von Thymohydrochinon ist konzentrationsabhängig. Bei Versuchen mit Fraktionen aus dem Humus (33) konnte in Sandkulturen beobachtet werden, dass die Aktivität von Aldolase im Spross von Roggenkeimpflanzen erhöht und die der Amylase erniedrigt wird. Die Aktivität der Saccharase in der Wurzel wird erhöht. Versuche mit verschiedenen Pflanzen und unter verschiedenen Bedingungen ergaben, dass mit steigenden Konzentrationen $\left(10^{-3}\right.$ bis $\left.10^{-5} \mathrm{~m}\right)$ an Thymohydrochinon auch der Gehalt an reduzierenden Zuckern und an Gesamtketosäuren zunahm (17). Ebenso wird der Gehalt an Brenztraubensäure erhöht, die ein wichtiges Zwischenprodukt für den Aufbau von Kohlenhydraten und für die Synthese von Aminosäuren ist. Thymohydrochinon zeigte in Bezug auf seine Einwirkung auf den Zuckerstoffwechsel eine Parallele mit Substanzen mit Wuchsstoffeigenschaften wie z.B. 2,4-Dichlorphenoxyessigsäure $(32,42)$, die ebenfalls den Gehalt an Zuckern in der Pflanze erhöht.

Pschenitschni (31) konnte mit infiltriertem Na-Humat ebenfalls die Erhöhung von reduzierenden Zucker in Keimpflanzen von Winterweizen zeigen.

Da der Citronensäurecyclus im Zusammenhang mit dem Atmungssystem der Pflanze steht, wurden verschiedene Säuren dieses Cyclus (17) besimmt, um weitere Erkenntnissse über die Einwirkung von Thymohydrochinon auf den Stoffwechsel der Pflanzen zu gewinnen. Hierzu wurde von der üblichen Versuchsanstellung mit Sandkulturen in Neubauerschalen oder mit Wasserkulturen abgegangen und 19 Tage alte in Nährlösungen gezogene Bohnenpflanzen in die Lösungen mit den zu prüfenden Substanzen in Konzentrationen von $10^{-3}$ Mol umgesetzt und nach $3 \mathrm{bzw}$. 7 Tagen die Blätter analysiert.

Thymohydrochinon erhöht den Gehalt an Brenztraubensäure, $a$-Ketoglutarsäure und den an Citronensäure, wie auch aus anderen Versuchen hervorgeht, recht deutlich. Der Gehalt an Äpfelsäure und Fumarsäure wird erniedrigt.

Mit Kulturen von isolierten Wurzeln $(18,16)$ und Messungen mit Blattrückständen (5) in der Warburg-Apparatur konnte gezeigt werden, dass Thymohydrochinon bei wachstumsfördernden Konzentrationen die Atmung erhöht.

Einen interessanten Versuch über den Einfluss von Humusstoffen auf die Sauerstoffaufnahme von Kartoffelblättern hat CHRISTEwA (41) durchgeführt. 
Tab. 6. Einfluss des organischen und anorganischen Anteils der Huminsäuren auf die Sauerstoffaufnahme der Kartoffelblätter.

\begin{tabular}{|c|c|c|c|c|c|}
\hline \multirow[t]{3}{*}{ infiltriert } & \multicolumn{3}{|c|}{$\begin{array}{c}\text { Sauerstoff in } \mathrm{mm}^{3} \text { aufgenommen nach } \\
10 \text { Minuten bei einer Einwaage von } \\
0,5 \mathrm{~g}\end{array}$} & \multirow[b]{3}{*}{ Mittel } & \multirow{3}{*}{$\begin{array}{l}\text { aufgenommen } \\
\text { Sauerstoff } \\
\text { in } \%\end{array}$} \\
\hline & \multicolumn{3}{|c|}{ Wiederholung } & & \\
\hline & 1. & 2. & 3. & & \\
\hline Na-Humat $0,001 \%$ & 78,6 & 112,7 & 105,5 & 98,9 & 140,4 \\
\hline dto. oxydiert mit $\mathrm{H}_{2} \mathrm{O}_{2}$ & 47,2 & 85,2 & 78,8 & 70,4 & 100,0 \\
\hline Na-Humat $0,001 \%$ & 100,0 & 115,1 & 104,2 & 106,4 & 147,6 \\
\hline Wasser & 70,0 & 78,2 & 68,3 & 72,2 & 100,0 \\
\hline
\end{tabular}

Die Sauerstoffaufnahme von Kartoffelblättern ist in Gegenwart von Natriumhumat erhöht. Da die Huminsäuren immer eine geringe Menge von Aschebestandteilen einschliessen, die einen Gehalt an Eisen aufweisen, oxydierte CHRISTEwA die Huminsäuren mit Wasserstoffperoxyd. Durch Vergleichsversuche konnte sie zeigen, dass die Wirkung von Natriumhumat auf die Sauerstoffaufnahme nur von organischem Anteil der Huminsäuren abhängt.

Thymohydrochinon beeinflusst ebenfalls die Wasseraufnahme und die Transpiration (23).

Es scheint unmöglich, aus diesen einzelnen Daten Aussagen über den Wirkungsmechanismus von Thymohydrochinon zu machen und diese mit der Wachstumsförderung in Beziehung zu bringen, da die Stoffwechselvorgänge mit ihren Wechsel-

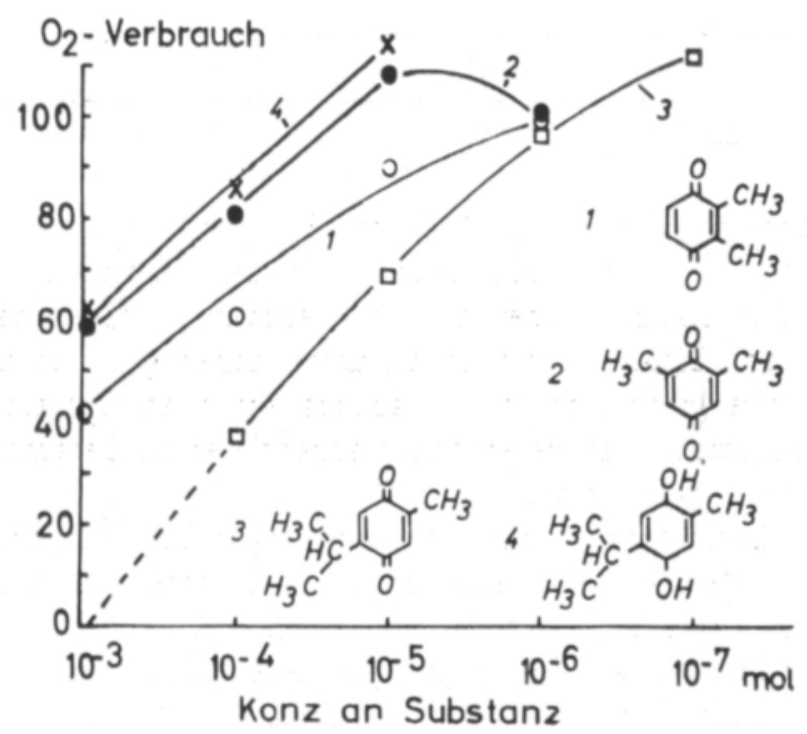

Abb. 4. $\mathrm{O}_{2}$-Verbrauch von verarmter Hefe unter dem Einfluss verschiedener Chinone, gemessen in $\mu \mathrm{l} / \mathrm{h}$, bezogen auf die gleich 100 gesetzte Kontrolle. 
wirkungen sehr kompliziert sind und aus dem Vergleich von Einzelvorgängen im Stoffwechsel nicht immer auf eine beobachtete Gesamterscheinung Schlüsse gezogen werden können. Eine Deutung der verschiedenen Befunde erfordert deshalb Untersuchungen an möglichst verschiedenen Organismen, wobei gerade solche an niederen Organismen wegen ihrer leichteren Handhabung bevorzugt herangezogen werden.

Um in den Mechanismus der Veränderungen des Stoffwechsels durch Chinone tiefer einzudringen, wurden neben den Untersuchungen an höheren Pflanzen auch solche an Hefe (Saccharomyces cerevisiae) (13) durchgeführt.

Die Atmungsmessungen in der Warburg-Apparatur ergaben beim Vergleich verschiedener Chinone und Hydrochinone eine Abhängigkeit der Wirkung von der Konstitution und der Konzentration, wie sie schon für die Wirkung auf das Pflanzenwachstum und den Ertrag gefunden wurde. So war z.B. der Sauerstoffverbrauch bei einer $10^{-5}$ molaren Konzentration von Thymohydrochinon erhöht. Bei stärkerer Verdünnung haben alle Substanzen keine Wirkung mehr auf den Sauerstoffverbrauch.

Die Bestimmung des Respirationsquotienten zeigt bei Konzentrationen von $10^{-3}$ bis $10^{-4} \mathrm{Mol}$ einiger der angewandten Chinone eine Verschiebung des Stoffwechsels zu Anaerobiose, d.h. sie regen die Gärung an, während bei anderen Chinonen kein Einfluss gefunden werden kann.

Glucoseverbrauch, Gehalt an Brenztraubensäure und Gesamtcarbonylverbindungen in verarmter Hefe nach 2 Stunden.

Thymohydrochinonkonzentration $10^{-5}$ molar.

$1000 \mathrm{mg}$ Glucose in der Aussenlösung (1\%).

\begin{tabular}{cccc}
\hline mg & Glucoseverbrauch & Brenztraubensäure & $\begin{array}{c}\text { Gesamtcarbonyl- } \\
\text { verbindungen }\end{array}$ \\
\hline Kontrolle & 480 & 8.0 & 11.5 \\
\hline Thymohydrochinon & 520 & 9.1 & 12.3
\end{tabular}

Bei einer Konzentration von $10^{-5} \mathrm{~m}$ an Thymohydrochinon, die die Sauerstoffaufnahme fördert, wird der Glucoseabbau in der Aussenlösung in den ersten Stunden erhöht. Die Befunde über den vermehrten Glucoseabbau bei den das Wachstum fördernden Konzentrationen stimmen daher gut mit dem gefundenen erhöhten Sauerstoffverbrauch bei den Warburgversuchen überein. Ebenso wird der Gehalt an Brenztraubensäure erhöht, während der an Gesamtcarbonylverbindungen nahezu unverändert bleibt.

Bei anaeroben Bedingungen unter Stickstoff ergab Thymohydrochinon in einer Konzentration von $10^{-5} \mathrm{~m}$ ebenfalls einen erhöhten Glucoseverbrauch in der Aussenlösung und eine vermehrte Bildung von Äthylalkohol. In höheren Konzentrationen war der Glucoseverbrauch vermindert und der Acetaldehydgehalt erhöht. Dieser Befund wird auf die Hemmung der Alkoholdehydrogenase zurückgeführt.

Es wurde weiterhin der Einfluss von Thymohydrochinon auf die Phosphorylierung (14) untersucht. 
In Konzentrationen von $10^{-3}$ bis $10^{-5} \mathrm{~m}$ wird die Phosphorylierung in $1 \%$ iger Glucoselösung durch die Zugabe von Thymohydrochinon in allen Fällęn vermindert. Da die Konzentration von $10^{-5} \mathrm{~m}$ den Sauerstoffverbrauch erhöht, geht aus den Werten für die Phosphorylierung auch gleichzeitig hervor, dass Thymohydrochinon in dieser Konzentration eine schwach entkoppelnde Wirkung besitzt. Zum Vergleich wurden Versuche über die Einwirkung auf die Phosphorylierung mit 2,4-Dinitrophenolionen 2,4-DNP in Konzentrationen von $10^{-3}-10^{-4} \mathrm{~m}$ angestellt, die nach Stickland (38) die Atmung erhöhen. Die Phosphorylierung wird durch diese Konzentrationen stark vermindert.

Da 2,4-DNP in Konzentrationen von $10^{-3}$ bis $10^{-4} \mathrm{~m}$ das Pflanzenwachstum nicht, dagegen Thymohydrochinon in einer Konzentration von $10^{-5} \mathrm{~m}$ das Pflazenwachstum fördert, scheint eine schwach entkoppelnde Wirkung für das Pflanzenwachstum von Bedeutung zu sein. Aus der Erhöhung der Atmung allein kann fernerhin nicht auf eine Förderung des Wachstums geschlossen werden, wie das Verhalten von 2,4 DNP zeigt. Die erhöhte Atmung ist also nicht die Ursache, sondern die Folge des Wachstums.

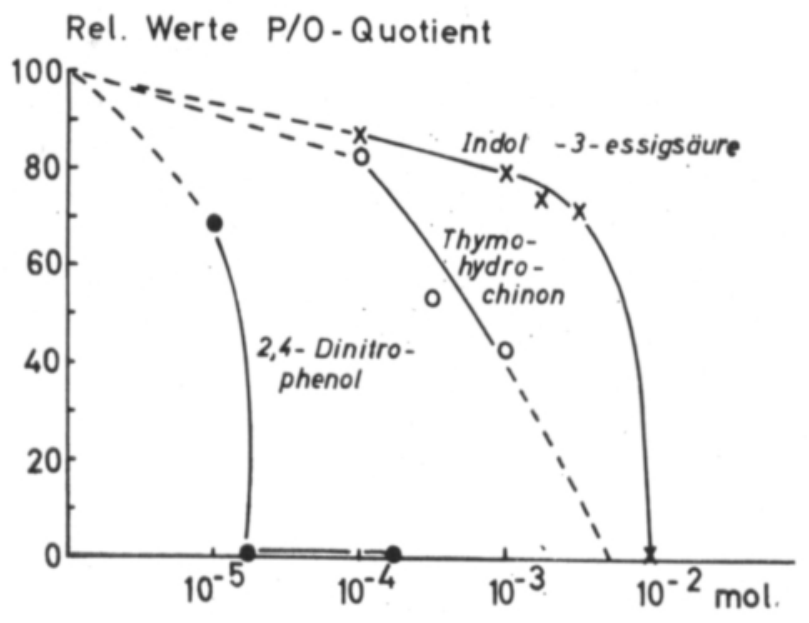

Abb. 5. Entkoppelung der oxydativen Phosphorylierung.

Die bisher aufgezeigten Untersuchungen über die Einwirkung von Thymohydrochinon und Thymochinon in wachstumsfördernden Konzentrationen auf den Stoffwechsel der Pflanzen ergaben Anhaltspunkte dafür, dass die Substanzen hierbei an zentraler Stelle im Stoffwechsel eingreifen (38). Wir haben daher die oxydative Phosphorylierung mit Mitochondrien aus Rattenleber und mit Fraktionen von Zellbestandteilen aus Brassicaarten untersucht (22).

In diese Untersuchungen wurden verschiedene Wuchsstoffe einbezogen, von denen zur Vereinfachung nur das Verhalten von Indol- 3-essigsäure erwähnt ist, und Substanzen, wie 2,4-DNP, die für eine starke Entkoppelung der oxydativen Phos- 
phorylierung bekannt sind. Als Modellsubstanz für Abbauprodukte von Lignin bzw. für Vorstufen von Huminsäuren werden die Werte von Thymohydrochinon angegeben.

Die Wirkungsweise der als pflanzliche Wuchsstoffe bekannten Verbindungen konnte bisher nicht mit einem einheitlichen Schema beschrieben werden. Die chemische Konstitution der natürlichen und synthetischen Wuchsstoffe ist meistens so verschieden, dass auf Grund dieser Eigenschaften keine überzeugende Erklärung für ihre Wirkung gefunden werden konnte. Nach den gesammelten Erfahrungen scheinen uns jedoch die allen Stoffen gemeinsame Wirkung auf die Atmung und die oxydative Phosphorylierung als Grundlage für einen allgemein gültigen Wirkungsmechanismus geeignet. In diesen Überlegungen lassen sich auch die im weiteren Sinne stoffwechselaktiven Substanzen einschliessen, zu denen Fraktionen aus dem Humus, Abbauprodukte des Lignins und chemisch ähnliche Substanzen zu zählen sind.

Alle diese Stoffe bewirken in Abhängigkeit von der Konzentration eine Erhöhung der Atmung und eine Entkoppelung der oxydativen Phosphorylierung.

Bei rascherem Wachstum müssen gewisse Zwischenprodukte des Stoffwechsels in verstärktem Masse zur Verfügung stehen, die aus den dafür verantwortlichen Prozessen entnommen werden. Eine Entkoppelung der oxydativen Phosphorylierung bewirkt einen höheren Gehalt an anorganischem Phosphat in der Zelle, wodurch Glykolyse und Säuerstoffwechsel erhöht werden, die an der Bildung von Zwischenprodukten zur Erzeugung von Pflanzensubstanz beteiligt sind. Die entkoppelnde Wirkung ist konzentrationsabhängig. Höhere Konzentrationen schädigen die für die Zwischenproduktbildung ablaufenden Stoffwechselvorgänge und führen zu Hemmungen des Wachstums. Wird die Konzentration verringert, so werden Glykolyse und Säurestoffwechsel nicht mehr gehemmt, sondern sogar gefördert. Es besteht jedoch die Möglichkeit, dass das für die Syntheseprozesse notwendige ATP immer noch in zu geringer Konzentration vorhanden ist, wodurch, wie z.B. beim 2,4-DNP trotz Erhöhung der Atmung das Wachstum gehemmt ist.

Nach diesen Ausführungen ist daher eine Verbindung umso eher als Wuchsstoff geeignet, als sie in einem relativ hohen und breiten Konzentrationsbereich schwach entkoppelt wie Indol-3-essigsäure. Andere Verbindungen, wie 2,4-DNP haben auf Grund eines weiten Konzentrationsbereichs mit stark entkoppelnder Wirkung einen herbiciden Effekt. Die Eigenschaften von Thymohydrochinon liegen dazwischen. Diese Erkenntnisse können im Prinzip auf alle stoffwechselaktiven Verbindungen übertragen werden. Ihre Wirkung kann jedoch in bestimmten Konzentrationen von Pflanze zu Pflanze verschieden sein. So ist z.B. auch bekannt, dass 2,4-Dichlorphenoxyessigsäure von Dikotyledonen leichter aufgenommen wird als von Monokotyledonen (7, 8). Die gefundenen Erhöhungen der Trockensubstanzerträge durch die Einwirkung von Thymohydrochinon, von Ligninabbauprodukten oder von chemisch ähnlichen Substanzen können auf Grund von Untersuchungen mit Mitochondrien über eine schwache Entkoppelung der oxydativen Phosphorylierung erklärt werden.

$\mathrm{Zu}$ Beginn der Ausführungen wurde erwähnt, dass die Einwirkung der organischen Stoffe aus dem Boden auf das Pflanzenwachstum ein sehr komplexes 
Problem ist. Die durchgeführten Untersuchungen tragen dazu bei, einige Veränderungen im Stoffwechsel als eine mehr oder minder direkte Folge der Einwirkungen der angewandten Substanzen zu erklären; es bestehen jedoch noch einige weitere Faktoren auf das Wachstum, die einmal im Zusammenhang mit der Verschiedenheit des Stoffwechsels von Pflanze zu Pflanze stehen, und zu anderen, die sich im Zusammenhang mit den Umweltbedingungen, in die die Pflanze hineingestellt ist, auswirken.

\section{I T E R A T U R}

(1) Beutelspacher, H. 1952. Physikalisch-chemische Beiträge zur Humusforschung. Z. Pflanzenern., Düng., Bodenk. 57: 57.

(2) Börner, H. 1956. Die Abgabe organischer Verbindungen aus den Karyopsen, Würzeln und Ernterückständen von Roggen (Secale cereale L.), Weizen (Triticum aestivum L.) und Gerste (Hordeum vulgare L.) und ihre Bedeutung bei der gegenseitigen Beeinflussung der höheren Pflanzen. Zeitschr. Biol. d. Pfl. 33: 35.

(3) Снaminade, R. 1955. Le potassium et la matière organique. Kalium - Symposium, S. 203. Bern.

(4) Christewa, L. A. 1957. Humusdüngemittel-Theorie und Praxis ihrer Anwendung (russ.), S. 101. Herausgegeben von der Landwirtsch. Hochsch. Cherson, verlegt von Univ. Charkow.

(5) Christewa, L. A. u.a. 1957. Die Rolle der Huminsäuren bei der Pflanzenernährung und die Humusdüngung (russ.). Arb. d. Dokutschajew-Instituts für Bodenk. der Akademie d. Wiss. d. UdSSR 38: 108.

(6) Dalton, J. D., Russel, J. \& Stieling, D. H. 1952. Effect of organic matter on phosphate availability. Soil. Sci. Sci. 73: 173.

(7) FANG, S. C. \& Butrs, J. S. 1954. Studies in plant metabolism. III. Absorption, translacation and metabolism of radioactive 2,4 - D in corn an wheat plants. Plant Physiol. 29: 56.

(8) Fang, S. C., Jarowski, E. G., Logan, A. V., Freed, V. H. \& Butts, J. S. 1951. The absorphtion of radioactive 2,4-dichlorphenoxyacetic acid and the translocation of $\mathrm{C}^{14}$ by bean plants. Arch. Biochem. Biophy. 32, Nr. 2.

(9) Flaig, W. 1958. Die Chemie organischer Stoffe im Boden und deren physiologische Wirkung. Verhandlungen d. II. und IV. Komission d. Internationalen Bodenkundlichen Gesellschaft. Vol. II. Hamburg.

(10) Flaig, W. 1958. Warum Modellsubstanzen? Ein Beitrag zur Chemie des Humus. Landbau Forsch. 9: 53.

(11) Flaig, W. \& Beutelspacher, H. 1951. Zur Kenntnis der Huminsäure. II. Elektronenmikroskopische Untersuchungen an natürlichen und synthetischen Humussäuren. Z. Pflanzenern., Düng., Bodenk. 52: 1.

(12) Flaig, W. \& Beutelspacher, H. 1954. Physikalische Chemie der Huminsäuren. Landbouwkund. Tijdschr. 66: 306 .

(13) Flatg, W. \& de JoNG, W. Untersuchungen über die Wirkung von p-Benzochinonderivaten auf den aeroben and anaeroben Stoffwechsel der Hefe (Saccharomyces cerevisiae). Archiv f. Mikrobiol. (im Druck).

(14) Flaig, W. \& de JoNG, W. Vergleich der Wirkung von Thymochinon und Thymohydrochinon mit der von 2,4-Dinitrophenol auf den Stoffewechel der Hefe (Saccharomyces cerevisiae). Ibid. (im Druck).

(15) Flaig, W. \& Maeder, H. Unveröffentlich.

(16) Flaig, W. \& Ruiz AmIL, M. Uber den Einfluss von Thymohydrochinon auf den Kohlenhydratstoffechsel von Dikotyledonen (span.). Anales de Edafologia y Fisilogia Vegetal (im Druck).

(17) Flaig, W. \& Ruiz AmIL, M. Uber den Einfluss von Thymohydrochinon auf den Saurestoffwechsel und die Atmung von Pflanzen (span.). Ibid. (im Druck). 
(18) Flaig, W. \& Saalbach, E. 1955. Zur Kenntniss der Huminsäuren. X. Uber den Einfluss des Thymolhydrochinons als Modellsubstanz von Vorstufen bezw. Abbauprodukten von Huminsäuren auf das Wurzelwachstum von Sommerweizen. Z. Pflanzenern., Düng., Bodenk. 71: 208.

(19) Flaig, W. \& SaAlbach, E. 1958. Beziehungen zwischen Kalium, Wasser und Boden. Kalium Symposium.

(20) Flaig, W. \& SaAlbach, E. 1959. Ủber den Einfluss von im Boden gerottetem Stroh auf das Wach. stum und die Nährstoffaufnahme von Roggenkeimpflanzen. Z. Pflanzenern., Düng., Bodenk. 87: 229.

(21) Flaig, W., Scharrer, K. \& Scholl, G. 1957. Zur Kenntnis der Humussäuren. XVI. Uber den Einfluss von Thymohydrochinon als Modellsubstanz von Humusstoffen auf die Aktivität verschiedener Enzyme des Roggens. Ibid. 76: 201.

(22) Flaig, W. \& Schmid, G. Über die Wirkung von Thymohydrochinon und Thymochinon im Vergleich mit Wuchsstoffen auf die oxydative Phosphorylierung. In Vorbereitung.

(23) Flatg, W. \& Scholl, G. Zur Kenntnis der Huminsäuren. XVIII. Uber den Einfluss von Thymohydrochinon auf den Wasserhaushalt lantwirtschaftlicher Kulturpflanzen (span.). Anales de Edafologia y Fisiologia Vegetal (im Druck).

(24) Flaig, W. \& Söchtig, H. 1959. Uber die Wechselwirkung zwischen Kaolinit and Polyanionen. Z. Pflanzenern., Düng., Bodenk. 87: 44.

(25) Fuller, W. H. \& Nielsen, D. R. 1957. The influence of straw and straw-fertilizercompost on the uptake of fertilizer phosphorus by plants. Proc. Soil Sci. Soc. Amer. 21: 278.

(26) HaGin, J. 1952. Influence of soil aggregation on plant growth. Soil Sci. 74: 471.

(27) Kononowa, M. M. \& PANkowa, N. A. 1950. Die Wirkung der Humusstoffe auf das Wachstum und die Entwicklung der Pflanzen (russ.). Berichte d. Akad. d. Wiss. d. UdSSR 73, Nr. 5.

(28) Maeder, H. 1960. Chemische und pflanzenphysiologische Untersuchungen mit Rottestroh. Diss., Giessen.

(29) Nishita, H., Kowalewsky, B. W. \& Larson, K. H. 1956. Influence of soil organique matter on mineral uptake by barley seedlings. Soil Sci. 82: 307 .

(30) Prat, S. \& PostPisil, F. 1959. Humic acids with C14. S. Biol. Plantarum (Praha) 1: 78.

(31) Pschenitschni, 1957. Humusdüngemittel-Theorie und Praxis ihrer Anwendung (russ.) S. 106 Herausgegeben von der Landwirtsch. Hochsch. Cherson, verlegt von Univ. Charkow.

(32) Rasmussen, L. W. 1947. The physiological action of 2,4-dichlorphenoxyacetic acid on dandelion (Taraxacum officinale). Plant Physiol. 22: 377.

(33) SaAlbach, E. 1955. Humusstoffe und Pflanzenwachstum. Landbauforsch. 5: 88.

(34) SaAlbach, E. 1958. Beitrag der Grundlagenforschung zum Problem der Strohdüngung. Landbauforsch. 8: 83 (1958); vergl. auch KNöSEL, D.: Pflanzenern., Düng., Bodenk. 80: 225 (1958), 85: 158 (1959).

(35) Sauerlandt, W. \& GrafF, O. 1955. Fragen der direkten Zufuhr von Stroh zum Ackerboden. Sonderdruck aus Bodenfruchtbarkeit» Oldenburg (Oldbg.).

(36) Sauerlandt, W. Scheffer, F. \& BAnse, H. J. 1957. Untersuchungen über organisch gebundenen Phosphor in verschiedener Böden. Z. Pflanzenern., Düng., Bodenk. 76: 244.

(37) Schachtschnabel, P. 1937. Aufnahme von nichtaustauschbarem Kali durch die Pflanzen. Bodenk. und Pflanzenern. 3: 107.

(38) StickLand, L. H. 1956. The pasteur effect in normal yeast and its inhibition by various agents. Biochem. J. 64: 503 .

(39) WeLte, E. 1954. Das Humusproblem - praktisch gesehen. I. Grundlagen der Humusersatzwirtschaft. Kalibriefe, Fachgebiet I, 7. Folge.

(40) Winter, A. G. 1955. Untersuchungen über Vorkommen and Bedeutung von antimikrobiellen und antiphytotischen Substanzen in natürlichen Boden. Z. Pflanzenern., Düng., Bodenk. 69: 224 .

(41) Winter, A. G. \& Schönbeck, F. 1959. Zum Wirkstoffkreislauf Pflanze - Tier - Boden. Ibid. 84, $1 / 3: 11$.

(42) Worp, D. J. 1951. Effects of non-lethal concentrations of 2,4-D on buckwheat. Plant Physiol. 26: 50 , 